\title{
Efecto de la Desnutrición Oculta Prenatal Sobre la Histología del Esplenio Callosal
}

\author{
Effect of Mild Protein Prenatal Malnutrition in the Callosal Splenium Histology
}

*Olivares, R.; " ${ }^{* *}$ Soto-Moyano, R.; ${ }^{* * *}$ Hernández, A.; ${ }^{* * * * *}$ Gil, J.; ${ }^{* * * *}$ Gimeno, M.; ${ }^{* * * *}$ Laborda, J. \& ****** Aboitiz, F.

OLIVARES, R.; SOTO-MOYANO, R.; HERNÁNDEZ, A.; GIL, J.; GIMENO, M.; LABORDA, J. \& ABOITIZ, F. Efecto de la desnutrición oculta prenatal sobre la histología del esplenio callosal. Int. J. Morphol., 25(4):723-727, 2007.

RESUMEN: Ratas malnutridas prenatalmente con una dieta isocalórica y baja en proteínas, presentaron un menor diámetro axonal promedio en el esplenio callosal que los animales control, tanto de las fibras mielínicas como amielínicas. También se observó una mayor densidad axonal promedio, con respecto a los controles. Estas observaciones sugieren que: 1) las conexiones cortico-corticales (interhemisféricas) son vulnerables a la malnutrición proteica; y 2) lo anterior tendría incidencia en la velocidad de conducción interhemisférica, en particular con lo que dice relación con las conexiones visuales.

PALABRAS CLAVE: Cuerpo Calloso; Esplenio; Malnutrición proteica prenatal; Axones; Fibras; Visión; Rata.

\section{INTRODUCCIÓN}

En ratas, la llamada desnutrición oculta prenatal es producida por una reducción de los contenidos de proteína en la dieta de las madres preñadas, desde un $25 \%$ a un $8 \%$ de caseína. Esta reducción es compensada calóricamente con un incremento de carbohidratos y grasa, lo cual produce pesos corporales y cerebrales normales en el neonato (Morgane et al., 1978; Resnick et al., 1982). Sin embargo, a largo plazo, esta condición produce alteraciones conductuales y en los desarrollos celular, neuroquímico y electrofisiológico, tales como reducción de la excitabilidad cortical producida por estimulación callosa, reducción en la capacidad de las sinapsis callosas para desarrollar sumaciones temporales, alteraciones en los niveles y liberación de noradrenalina cortical y déficit en pruebas de baja complejidad (Stern et al., 1975; Stern et al., 1976; SotoMoyano et al., 1998; Soto-Moyano et al., 1999). La rehabilitación postnatal con una dieta alta en proteína restaura algunos de estos déficits (Soto-Moyano et al., 1998; SotoMoyano et al., 1999).

Estudios previos han demostrado que a los 45 a 52 días de edad, ratas malnutridas prenatalmente y durante la lactancia con una dieta isocalórica e hipoproteica, presenta- ron un cuerpo calloso más pequeño que las ratas control. En cambio aquellos animales rehabilitados durante la lactancia con una dieta alta en proteínas, mostraron normalidad en el peso cerebral y en el desarrollo de los tercios medio y posterior del cuerpo calloso. Sin embargo, el tercio anterior del calloso, que conecta áreas frontales, es particularmente afectado por este tipo de malnutrición, a pesar de una rehabilitación dietaria durante la vida postnatal (Olivares et al., 2002).

Dada la importancia de este tracto en las conexiones cortico-corticales (interhemisféricas) y la evidencia anteriormente señalada, se consideró de interés el estudiar los posibles efectos de una desnutrición oculta prenatal sobre la histología del cuerpo calloso (densidad y diámetros fibrilares). Aunque las regiones posteriores del cuerpo calloso parecen conservar su morfología gruesa en condiciones de desnutrición oculta, es posible que existan alteraciones microscópicas que escapan a un análisis macroscópico. Por esta razón, hemos decidido estudiar el quinto posterior del cuerpo calloso (esplenio). En el humano y en el macaco, este segmento calloso contiene, en el extremo posterior, fibras visuales provenientes de la corteza occipital (Pandya \& Seltzer 1986; Witelson, 1989; Aboitiz et al., 1992).

* Departamento Ciencias Biológicas Animales, Facultad Ciencias Veterinarias y Pecuarias, Universidad de Chile.

** Laboratorio de Hormonas y Receptores, Instituto de Nutrición y Tecnología de los Alimentos, Universidad de Chile.

*** Departamento de Biología, Facultad de Química y Biología, Universidad de Santiago de Chile.

***** Departamento de Anatomía, Embriología y Genética, Facultad de Veterinaria, Universidad de Zaragoza, España.

******Laboratorio de Neurobiología, Centro de Investigaciones Médicas, Pontificia Universidad Católica de Chile.

Financiamiento: Centro de Neurociencias Integradas, iniciativa científica Milenio Fondecyt 1030729. 


\section{MATERIAL Y MÉTODO}

Se utilizaron tres ratas adultas (60-62 días de edad), de la cepa Sprague-Dawley, provenientes de camadas ajustadas a 8 crías (4 machos y 4 hembras), cuyas madres recibieron durante la gestación una dieta hipoproteica-isocalórica (8\% de caseína), y después del nacimiento fueron criadas por nodrizas controles hasta el destete. Como controles se utilizaron tres ratas, cuyas madres recibieron durante la gestación y hasta el destete de las crías, una dieta isoproteicaisocalórica ( $25 \%$ de caseína).

Los animales fueron anestesiados con uretano $(1.5 \mathrm{~g} /$ $\mathrm{Kg}$ i.p.) y perfundidos transcardialmente con solución de Karnovsky (glutaraldehido $1.5 \%$ y paraformolaldehido $0.8 \%$ ) para, posteriormente, extraer los encéfalos y disecar los esplenios callosales (quinto posterior del cuerpo calloso). Estos últimos fueron fijados en glutaraldehido al $2.5 \%$ por 8 horas, a $4^{\circ} \mathrm{C}$, luego lavados en buffer fosfato de sodio $0.1 \mathrm{M} \mathrm{pH} 7.3$, postfijados en tetróxido de osmio al $1 \%$, por 1 hora a temperatura ambiente $\mathrm{y}$, finalmente, fueron incluidos en epon 812 .

Se realizaron cortes semifinos de $1 \mathrm{~mm}$, teñidos con azul de toluidina en borato de sodio al $1 \%$, para precisar la zona del esplenio (región posterior) donde se realizaría el conteo (expresado en número de axones por $\mathrm{mm}^{2}$ ) y la medición de los diámetros de las fibras callosales mielínicas y amielínicas (expresado en $\mu \mathrm{m}$ ). Luego de esto, se realizaron cortes finos de $60 \mathrm{~nm}$ montados en grillas de 200Mesh, los que fueron contrastados con acetato de uranilo en metanol al $4 \%$, por 1 minuto y citrato de plomo, por 5 minutos, para su visualización a microscopía electrónica de transmisión (MET, Zeiss Modelo EM 109). La observación se realizó a una magnificación adecuada para una clara visualización de los axones mielínicos y amielínicos (7.000X).

Del esplenio correspondiente a cada animal, se fotografiaron 5 campos $(27.000 \times 23.000 \mu \mathrm{m})$, los que fueron revelados en papel $(9 \times 11 \mathrm{~cm})$, digitalizados y analizados con el programa Scion Image, a una magnificación total de 8.800X. El conteo fibrilar se realizó excluyendo los axones que tocaban los bordes inferior e izquierdo de la fotografía (Gundersen, 1977). Se contabilizaron un promedio de 184 fibras por campo. Con respecto a las fibras mielínicas, se consideró el diámetro interno (excluyendo la vaina de mielina).

Los valores de densidad y diámetro fibrilar promedios de las fibras mielínicas y amielínicas, fueron comparados entre los animales tratados y controles mediante la prueba de $t$ de Student.

\section{RESULTADOS}

Diámetro fibrilar promedio (Fig. 1). El grupo desnutrido $(\mathrm{n}=3)$, presentó un diámetro promedio menor de fibras mielínicas que el grupo control $(n=3)(0.40 \pm 0.04 \mu \mathrm{m}$ vs. $0.52 \pm 0.04 \mu \mathrm{m} ; \mathrm{p}<0.05)$.

El grupo desnutrido también presentó un diámetro promedio menor de fibras amielínicas que el grupo control $(0.17 \pm 0.02 \mu \mathrm{m}$ vs. $0.22 \pm 0.03 \mu \mathrm{m} ; \mathrm{p}<0.05)$.

Densidad fibrilar promedio (Fig. 2). El grupo desnutrido presentó una mayor densidad de fibras mielínicas que el grupo control $\left(0.16 \pm 0.04\right.$ axones $/ \mathrm{mm}^{2}$ vs. $0.1 \pm$ 0.02 axones $/ \mathrm{mm}^{2}$ ). El grupo desnutrido también presentó una mayor densidad de fibras amielínicas $(0.20 \pm 0.08$ axones/ $\mathrm{mm}^{2}$ vs. $0.13 \pm 0.03$ axones $/ \mathrm{mm}^{2}$ ). Sin embargo, estas diferencias no alcanzaron significancia estadística.

Las Figs. 3 y 4 muestran las fibras mielínicas y amielínicas del esplenio callosal de animales de los grupos control y desnutrido.

\section{DIAMETRO FIBRILAR PROMEDIO}

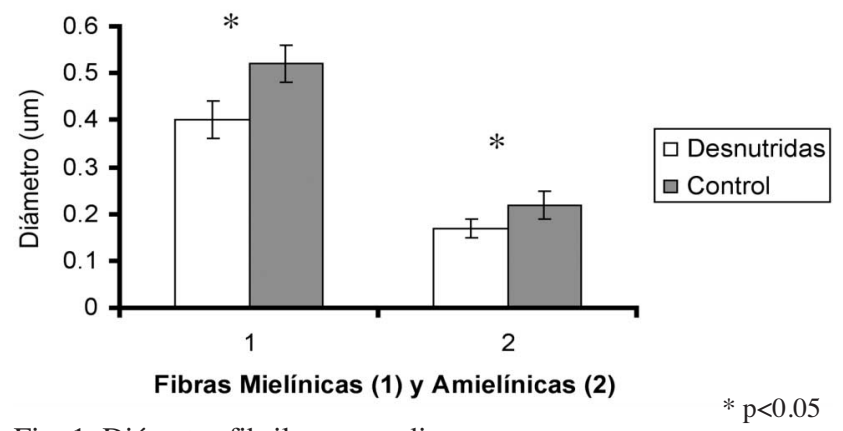

Fig. 1. Diámetro fibrilar promedio.

\section{DENSIDAD FIBRILAR PROMEDIO}

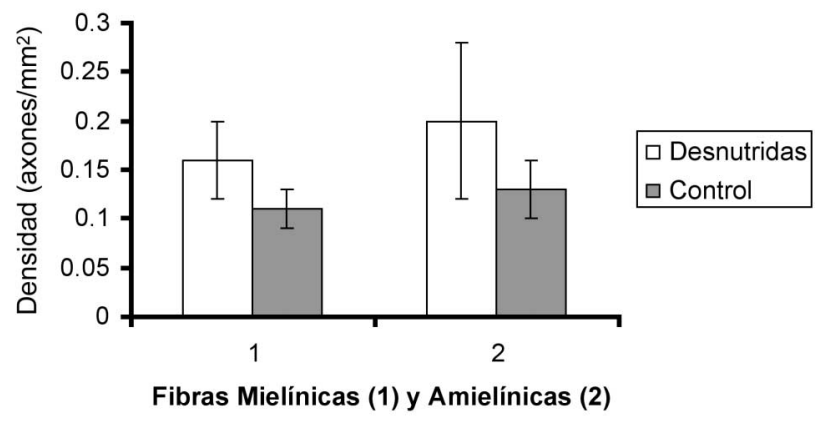

Fig. 2. Densidad fibrilar promedio. 


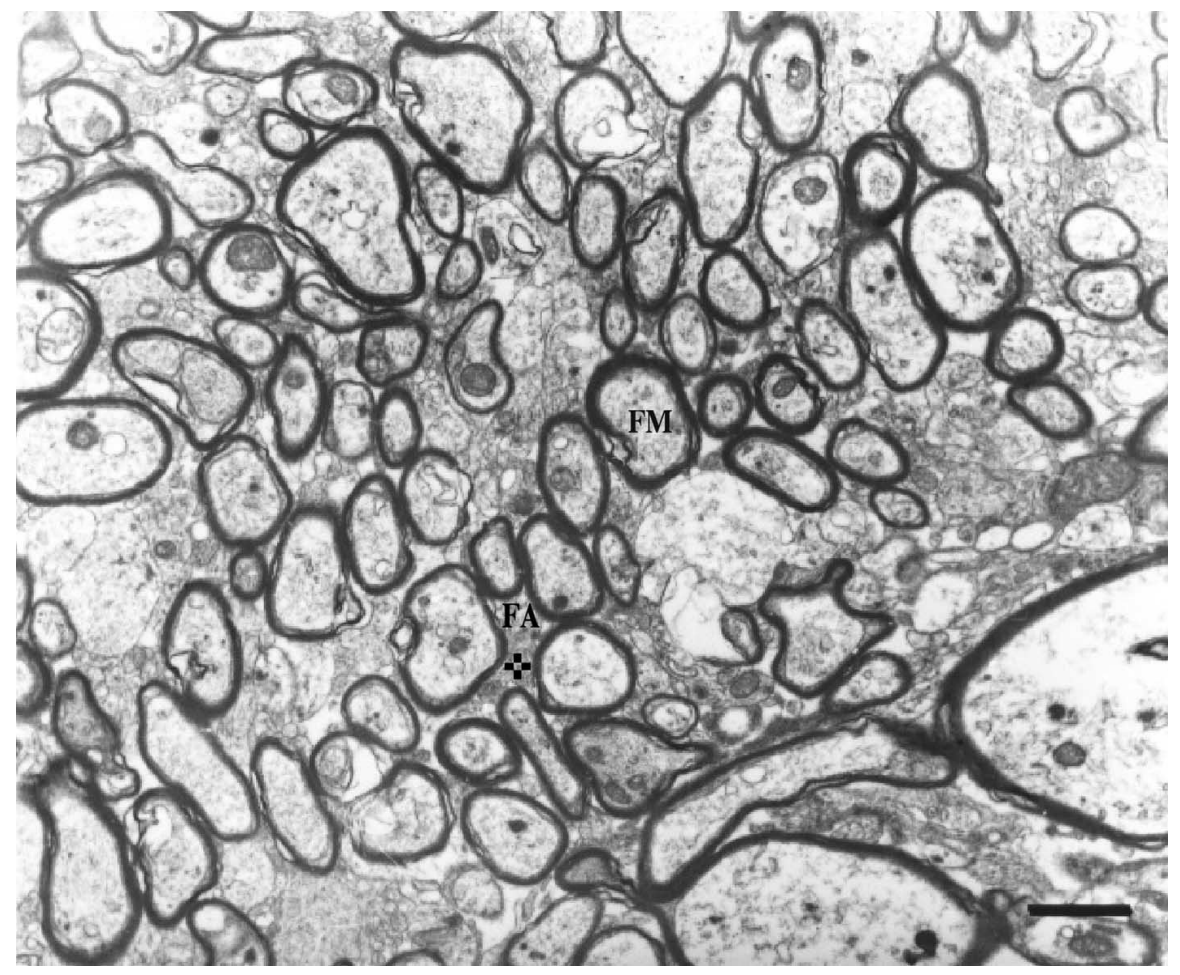

Fig. 3. Microfotografía de esplenio callosal de animal control $($ barra $=1 \mu \mathrm{m})$. $\mathrm{fm}=$ fibra mielínica; fa = fibra amielínica.

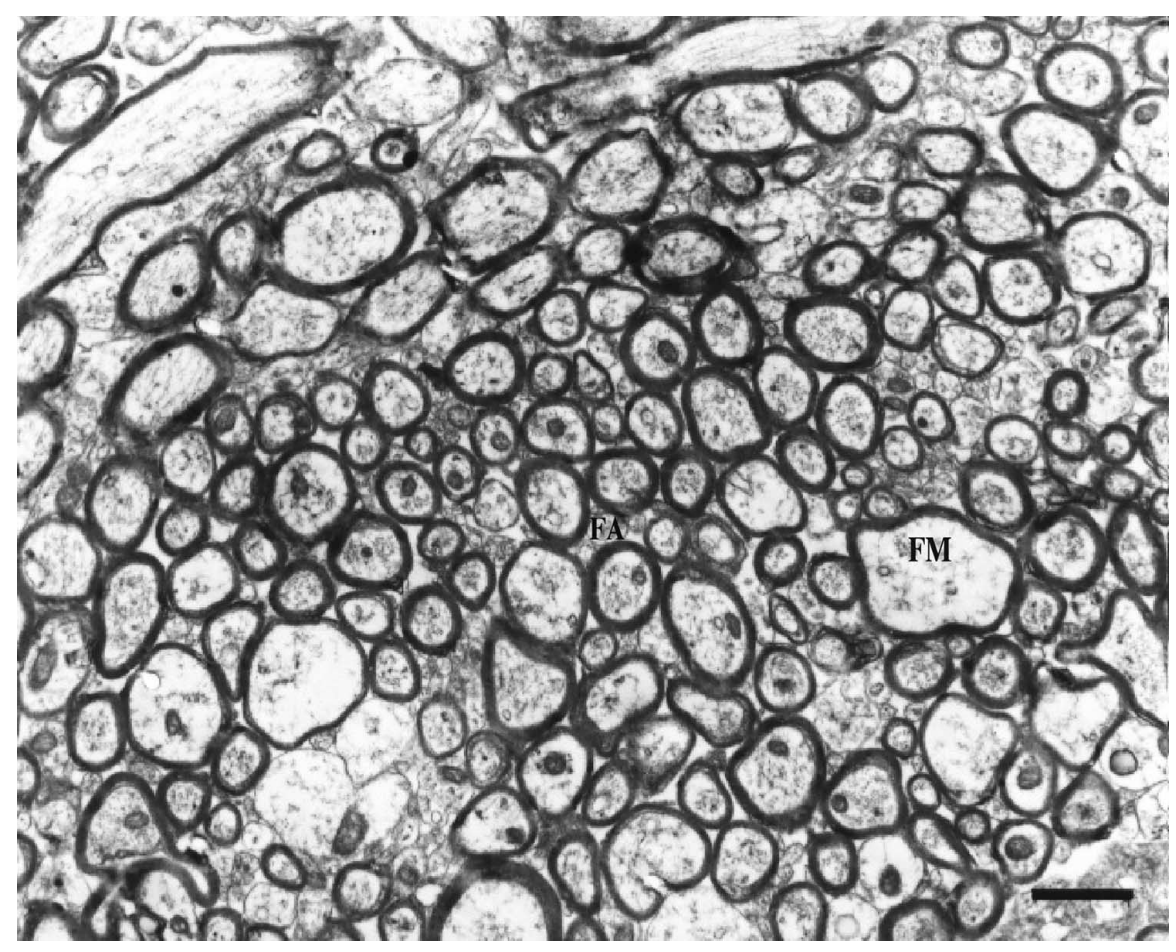

Fig. 4. Microfotografía de esplenio callosal de animal desnutrido (barra $=1 \mu \mathrm{m})$. $\mathrm{fm}=$ fibra mielínica; fa = fibra amielínica.

\section{DISCUSIÓN}

El modelo de la desnutrición oculta prenatal fue originalmente descrito en ratas (Morgane et al.; Resnick et al.) y es de gran interés porque no produce diferencias en los pesos corporales y cerebrales al nacimiento, al compararlo con ratas normales. Sin embargo, subsisten diferencias, en los desarrollos neuronal y electrofisiológico, entre los animales controles y los malnutridos. En particular en estas ratas, los elevados niveles de noradrenalina y la liberación de noradrenalina inducida por potasio (Stern et al., 1975; Resnick et al.; Soto-Moyano et al., 1998), pueden afectar fenómenos tales, como sinaptogénesis, muerte celular y retracción sináptica durante el desarrollo cerebral temprano.

Ratas de 8 días de edad, hijas de madres sometidas a desnutrición oculta prenatal, presentaron una densidad tres veces mayor de adrenoreceptores a $2 \mathrm{c}$ en la neocorteza, que las ratas control. A los 60 días de edad, la densidad de estos receptores aún se mantuvo elevada, los animales no fueron capaces de mantener una potenciación de largo plazo en la neocorteza y presentaron un bajo rendimiento en pruebas de memoria visoespacial (Soto-Moyano et al., 2005). Además, las dendritas apicales de las células piramidales son más cortas y sus espinas dendríticas menos densas, en estos animales que en los controles (West \& Kemper, 1976), particularmente en células ubicadas en las capas II, III y V (Diaz-Cintra et al., 1990). Electrofisiológicamente, se ha observado una reducción en la actividad espontánea neuronal cortical (Stern et al., 1983) y en la excitabilidad neuronal cortical (Soto-Moyano et al., 1981 and 1998), a pesar de una rehabilitación nutricional postnacimiento. 
Se ha evidenciado que la transmisión callosal en el recién nacido se ve afectada por este tipo de malnutrición, produciendo un aumento en la latencia de la respuesta evocada transcallosal, la cual tiende a desaparecer después del destete (Morgane et al., 1978) y una alteración en los mecanismos sinápticos de la conexiones callosales (Soto-Moyano et al., 1999).

Aunque en roedores no se ha desarrollado un mapa tan exhaustivo, evidencias sugieren que el calloso posterior aloja fibras visuales, las cuales no obstante se distribuyen en forma más dispersa que en el macaco (Kim et al., 1996).
Los resultados del presente trabajo indican que la velocidad de transmisión interhemisférica debe estar afectada por la desnutrición oculta, en particular lo que dice relación con las conexiones visuales, lo cual apoya los resultados previos de Soto-Moyano et al. (1998). Para determinar si existe una diferencia en el número de axones callosos, es necesario realizar un muestreo más amplio, como también medir las respectivas áreas del cuerpo calloso.

Agradecimientos: Al Dr. Mauricio Canals por su asesoría computacional.

OLIVARES, R.; SOTO-MOYANO, R.; HERNÁNDEZ,A.; GIL, J.; GIMENO, M.; LABORDA, J. \& ABOITIZ, F. Effect of mild protein prenatal malnutrition in the callosal splenium histology. Int. J. Morphol., 25(4):723-727, 2007.

SUMMARY: Adult rats malnourished prenatally with a low-protein, isocaloric diet showed smaller median fiber diameter of myelinated and unmyelinated fibers and a higher axonal density in the callosal splenium than controls. These findings suggest (i) that cortico-cortical (interhemispheric) connections are vulnerable to protein malnutrition; and (ii) this may affect interhemispheric conduction velocity, particularly in visual connections.

KEY WORDS: Corpus callosum; Splenium; Prenatal Protein Malnutrition; Axons; Fibers; Vision; Rat.

\section{REFERENCIAS BIBLIOGRÁFICAS}

Aboitiz, F.; Scheibel, A.B.; Fisher, R. S. \& Zaidel, E. Fiber composition of the human corpus callosum. Brain Res., 598:143-53 1992.

Diaz-Cintra, S.; Cintra, L.; Ortega, A.; Kemper, T. \& Morgane, P. J. Effects of protein deprivation on piramidal cells of the visual cortex in rats of three ages groups. $J$. Com. Neurol., 292:117-26, 1990.

Gundersen, H. J. Notes on the estimation of the numerical density of arbitrary profiles. The edge effect. J.Microsc., 111:219-23, 1977.

Kim, J. H. Y.; Ellman, A. \& Juraska, J. M. A re-examination of sex differences in axon density and number in the splenium of the rat corpus callosum. Brain Res., 740:4757, 1996.

Morgane, P. J.; Miller, M.; Kemper, T.; Stern, W.; Forbes, W.; Hall, R.; Bronzino, J.; Kissane J.; Hawrylewoiz, E. $\&$ Resnick, O. The effects of protein malnutrition on the developing central nervous system of the rat. Neurosc. Biobehav. Rev., 2:137-230, 1978.

Olivares, R.; Soto-Moyano, R.; Hernández, A. \& Aboitiz F. La malnutrición prenatal proteica leve afecta el desarro- llo del cuerpo calloso anterior. Rev. Chil. Anat., 20:15963, 2002.

Pandya D. N. \& Seltzer, B. In: Two Hemispheres-One Brain. Functions of the corpus callosum. Eds. Leporé F., Ptito M. \& Jasper H. H. (Liss, New York), 1986. pp 47-73.

Resnick, O.; Morgane, P.J.; Hasson, R. \& Miller, M. Overt and hidden forms of cronic malnutrition in the rat and their relevance to man. Neurosc. Biobehav. Rev., 6:5575,1982 .

Soto-Moyano, R.; Ruiz, S.; Carillo, R. \& Hernández, A. Effect of prenatal malnutrition on cortical reactivity of the rat parietal association area. Int. J. Neurosc., 13:99102, 1981.

Soto-Moyano, R.; Alarcon, S.; Belmar, J.; Kusch, P.; Pérez, H.; Ruiz, S. \& Hernández, A. Prenatal protein restriction alters synaptic mechanisms of callosal connections in the rat visual cortex. Int. J. Dev. Neurosc., 16:75-84, 1998.

Soto-Moyano, R.; Fernández, V.; Sanhueza, M.; Belmar, J.; Kusch, C.; Pérez, H.; Ruiz, S. \& Hernández, A. Effects of mild protein prenatal malnutrition and subsequent 
postnatal nutritional rehabilitation on noradrenaline release and neuronal density in the rat occipital cortex. Dev. Brain Res., 116:51-8, 1999.

Soto-Moyano, R.: Valladares, L.; Sierralta,W.; Pérez, H.; Mondaca, M.; Fernández, V.; Burgos, H. \& Hernández, A. Mild prenatal protein malnutrition increases a2Cadrenoceptor density in the cerebral cortex during postnatal life and impairs neocortical long-term potentiation and visuo-spatial performance in rats. J. of Neurochemistry, 93:1099-109, 2005.

Stern, W. C.; Miller, M.; Forbes, W. B.; Morgane, P. J. \& Resnick, O. Ontogeny of the levels of biogenic amines in various parts of the brain and peripherical tissues in normal and malnourished rats. Exp. Neurol., 49:314-26, 1975.

Stern, W. C.; Resnick, O. \& Morgane, P. J. Developmental malnutrition in rats: Acquisition and long term retention of a single alternation discrimination. Nutr. Rep. Internat., 14:475-83, 1976.

Stern, W. C.; Pugh, W. W.; Johnson, A. \& Morgane, P. J. Spontaneus forebrain neuronal activity in developmentally protein malnourished rats. Brain Res., 285:95-8, 1983.

West, C. D. \& Kemper, T. L. The effects of low protein diet on the anatomical development of the rat brain. Brain Res., 107:221-37, 1976.

Witelson S.F. Handedness and sex differences in the isthmus and genu the human corpus callosum: a postmortem morphological study. Brain, 112:799-835, 1989.
Dirección para correspondencia: Dr. Ricardo Olivares Pérez-Montt Depto. de Ciencias Biológicas Animales Facultad de Ciencias Veterinarias y Pecuarias Universidad de Chile Santiago - CHILE

Email: rolivare@uchile.cl

Recibido : 13-06-2007

Aceptado: 26-08-2007 
